\title{
Self-Calibration of Extremely Unstable Sensor Arrays
}

\author{
G. Magna ${ }^{1}$, C. Di Natale, E. Martinelli \\ Department of Electronics Engineering, University of Rome "Tor Vergata", via del Politecnico 1,00133, \\ Rome, Italy \\ Corresponding author martinelli@ing.uniroma2.it
}

\begin{abstract}
Classification models accomplish the recognition of unknown samples relying on a training set of data. However, since sensors are usually unstable over the long period, perpetuating the use of a same sensor array can make outdated the classification model. In these situations, if the array still maintains the capability of discriminating classes, periodical calibrations are sufficient to preserve performances. Conversely, sensor failures or malfunctioning could compromise current and further classification models; hence defective sensors have to be removed and, in case, replaced. This work investigates the implementation of a Self-Calibration algorithm aimed at detecting the occurrence of dramatic changes in the behavior of sensors. Once an anomaly is detected, the model decides either to keep or to remove the affected sensor. In the latter situation, a sensor replica is included in the array. Eventually, a model for the whole array might be trained in a totally unsupervised way and the algorithm utilizes the subset of the array still functioning to recalibrate the whole array [1]. Results show that, in terms of classification rate, the non-malfunctioning and malfunctioning scenarios are alike in case of self-calibrated models.
\end{abstract}

Key words: calibration, drift, fault, gas sensor array

\section{Introduction}

This work illustrates an algorithm designed for protracted experiments aimed at chemical patterns recognition with gas sensor arrays. Among the complications, likely to be found in long lasting experiments, drift is the preeminent one. Drift is defined as the change of the sensor responses to the same stimulus over a long period. The consequence of drift is the gradually decrease of the performance of any classifier applied to the sensor array data.

Frequent exposure to reference gases [2] and adaptive models [3] have been often suggested to remediate to the presence of drift. However, most of these techniques may fail if one or few sensors become suddenly non-selective or highly noised. This work illustrates an unsupervised calibration procedure, hereinafter Self-Calibration, to deal with both drift and the appearance of sudden anomalies in sensors.

\section{Experimental and results}

The Self-Calibration algorithm detects malfunctioning sensors. Once an anomalous sensor behavior is detected, the algorithm decides if the sensor might be still useful, and then leaves it in the array (model reboot), or if it is no longer viable and then the sensor is replaced (Fig 1A). In both cases a new calibration is performed (Fig. 1B). A reduced model is built with only the reliable sensors, samples are collected and the class predictions of the minimal array are used as labels. Once a sufficient number of training samples is collected, a new model can be calculated. Fig. 2 shows the algorithm timeline. The SelfCalibration procedure has been investigated in the case of an ensemble of adaptive classifiers, called Cooperative Classifier [4]. The extension to more standard classifiers can be obtained with minimal adjustments.

The properties of the algorithm have been studied with a dataset made of 60 training and 240 test samples. Data were obtained in an experiment with an array made of 4 replicas of 4 different metal oxide semiconductor gas sensors exposed to 3 different classes. The initial array was made with only 4 sensors and other replicas were left for sensor replacing. Permanent or temporal sensor anomalies have been simulated (Figure $3 \mathrm{~A}$ and $3 \mathrm{~B}$ respectively). The algorithm performance is compared with standard classifiers such as kNN and PLS-DA. Results show that the decision-making process enables to maintain 
the classification rates close to the nonperturbed case in all the considered conditions.

A)

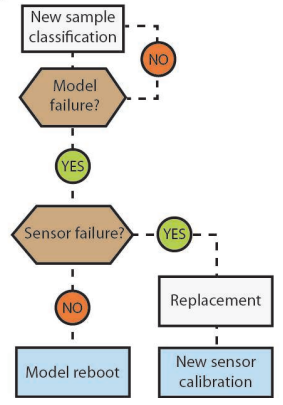

B)

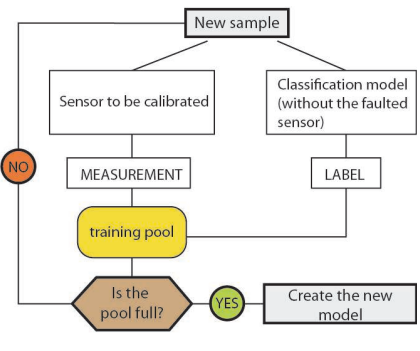

Fig. 1. Flow chart of the anomalies detection and the decision step (panel A). Self-Calibration of a new classification model (Panel B).

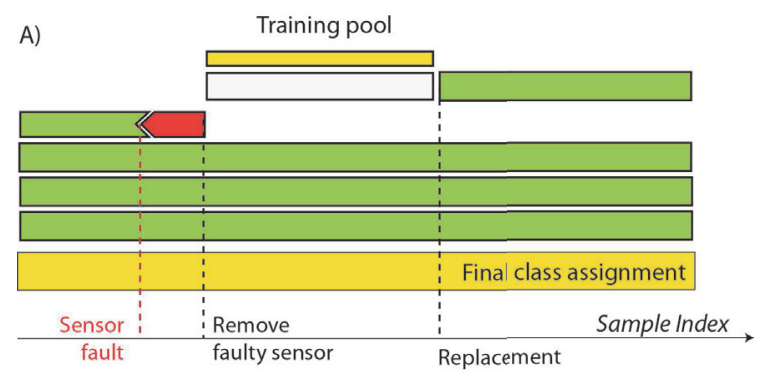

B)

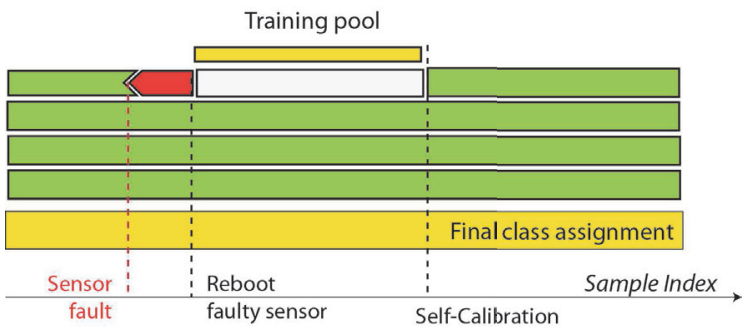

Fig. 2. A) Replacement and B) Reboot experimental timeline. In the second case, the anomalous sensor is re-included in the sensor array.

\section{References}

[1] M. A. Carpenter, S. Mathur, A. Kolmakov, Metal oxide nanomaterials for chemical sensors. Springer Science \& Business Media (Eds.2012).

[2] J. E. Haugen, O. Tomic, K. Kvaal, A calibration method for handling the temporal drift of solid state gas-sensors, Analytica chimica acta, 407(12), 23-39, (2000); doi: 10.1016/S00032670(99)00784-9

[3] H. Kaneko, K. Funatsu, Classification of the degradation of soft sensor models and discussion on adaptive models. AIChE Journal, 59(7), 23392347, (2013); doi: 10.1002/aic.14006

[4] E. Martinelli, G. Magna, A. Vergara, C. Di Natale, C. Cooperative classifiers for reconfigurable
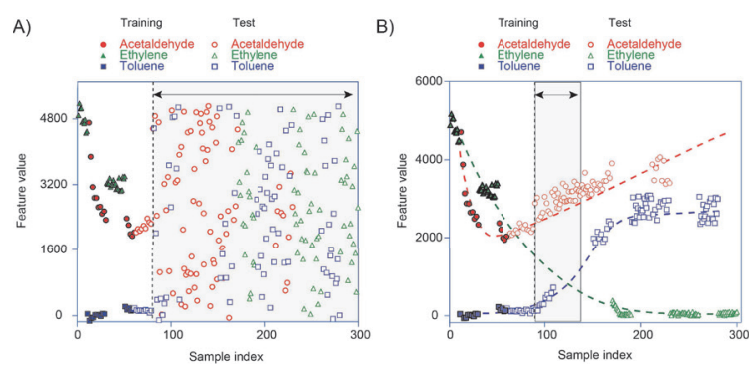

Fig. 3. A) Permanent and B) temporal anomalies induced in one sensor in the array.

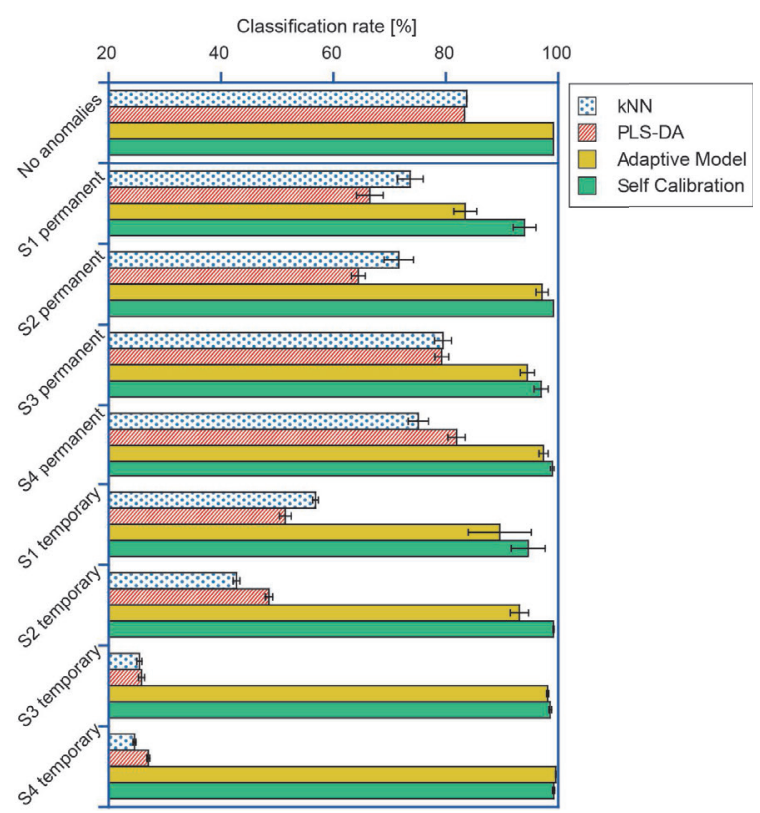

Fig. 4. Results obtained after inducing the two faulty conditions (permanent and temporary) to each sensor of the initial array (S1, S2, S3, and S4). Error bars are calculated with 100 simulations for each case.

sensor arrays, Sensors and Actuators B:

Chemical, 199, 83-92, (2014); doi:

10.1016/j.snb.2014.03.070 